\title{
TRICHODERMA SPP ANTAGONISMUS ZU PHYTOPATHOGENEN VERURSACHT MANIOKWURZELFÄULE IN ALAGOAS
}

\section{REVIEW ARTICLE}

SILVA, Izael Oliveira ${ }^{1}$

AMORIM, Edna Peixoto da Rocha ${ }^{2}$

JUNIOR, Nelson Augusto Nascimento ${ }^{3}$

PEIXINHO, Georgia Souza ${ }^{4}$

CARNAÚBA, Juliana Paiva ${ }^{5}$

NETO, Vicente Ferreira de Araujo ${ }^{6}$

SILVA, Izael Oliveira. Et al. Trichoderma spp Antagonismus zu Phytopathogenen verursacht Maniokwurzelfäule in Alagoas. Revista Científica Multidisciplinar Núcleo do Conhecimento. Jahrgang 05, Ed. 06, Vol. 12, S. 149-177. Juni 2020. ISSN: 2448-

\footnotetext{
${ }_{1}^{1}$ Promotion in Pflanzenschutz, Master in Plant Production Master es Master, Bachelor es degree und Bachelor es Degree in Biological Sciences.

2 Promotion in Agronomy (Landwirtschaft). Master-Abschluss in Phytopathologie. Spezialisierung in Didaktik für Hochschulbildung. Abschluss in Agronomy.

${ }^{3}$ Promotion in Pflanzenschutz. Master-Abschluss in Agronomie (Bewässerung und Entwässerung). Abschluss in Agronomy.

${ }^{4}$ Master in Irrigated Horticulture. Abschluss in Agronomy.

$5 \mathrm{PhD}$ in Phytopathologie. Master in Agronomy (Pflanzenproduktion und Pflanzenschutz). Verbesserung der Verbesserung der biologischen Wissenschaften. Abschluss in Agronomy.

${ }^{6}$ Abschluss in Agronomy.
} 
0959, Zugriffslink: https://www.nucleodoconhecimento.com.br/agronomiede/trichoderma-antagonismus

\section{ZUSAMMENFASSUNG}

Einige sind Phytopathogene, die die Produktion von Maniava (Manihot esculata Crants) begrenzen, die Fäulnis der Wurzeln sind, mit denen sie den größten Verlust in der Ernte heiraten und 70\% erreichen. Diese Kultur ist für die Region Nordostbrasilien von großem Interesse und verursacht große Verluste. Die Schwierigkeiten im Management haben die Suche nach Alternativen geleitet, unter denen Biocontroller signifikante Ergebnisse gezeigt haben. Diese Literaturrezension zielte darauf ab, Informationen über die Fäulnis der Maniokwurzel cv Rosinha, die wichtigsten Phytopathogene und antagonistische Aktivitäten der Trichodermie bei der Bekämpfung von Krankheiten im Bundesstaat Alagoas zu sammeln. Eine bibliographische Umfrage wurde in den Datenbanken Scielo, Google Acad'mico, Capes Portal (Thesen und Dissertationen), Zeitschriften und Büchern durchgeführt. Um Informationen über: Wurzelfäule von Tischmaniok, Trichoderma spp., als Biokontrollmittel von Phytopathogenen zu erhalten.

Schlagworte: Rohrwurzel, biologische Kontrolle, Phytopathologie.

\section{EINFÜHRUNG}

Aufgrund seines Nährwerts zeichnet sich Manihot esculenta Crantz in Brasilien und weltweit durch seine Vielseitigkeit durch, da es sowohl in tierischen als auch in menschlichen Lebensmitteln eingesetzt und in der Industrie weit verbreitet ist. Die Ernte übt eine bedeutende Unterstützung für die Bevölkerung mit geringer Kaufkraft aus, weil sie nicht nur eine nahrhafte Quelle ist, sondern auch ein Einkommensgenerator in der Familienlandwirtschaft ist (NOTARO et al. 2013).

In der Welt, der Verbrauch von Maniok und seine Derivate sind an sechster Stelle in der Rangliste sind Sojabohnen, Weizen, Reis, Mais und Kartoffeln die Kulturen, die die 
ersten Positionen nach fao Daten (2012). Brasilien ist mit einer geschätzten Ernte von 25 Millionen Tonnen für 2016 der viertgrößte Produzent der Welt (IBGE, 2015).

Von großer Vielseitigkeit ist die Ernte voll ausgereicht, nicht nur der Luftteil (Blätter und Stiele), sondern auch die Wurzel. Die Blätter haben ihre Verwendung in menschlichen (Ergänzung) und tierischen (zerkleinerten) Lebensmitteln. Die Stiele, in Tierfutter, in Form von Sistilladen und Heu und noch in Natura. Die Wurzel, in der menschlichen Nahrung, Tier und auch von Industrien, vor allem Mehl, Stärke, biologisch abbaubare Kunststoffe, Textilprodukte und Biokraftstoff (CONAB, 2013).

Die Cassava-Kultur steht vor vielen Herausforderungen, darunter phytosanitäre Probleme (NASCIMENTO J'NIOR, 2015). Mehrere Krankheiten wurden bereits in der Kultur berichtet, darunter Wurzelfäule, deren Erreger Lasiodiplodia sp., Pythium sp., Roselinia sp., Fusarium sp., Neoscytalidium lignicola, Phytophthora drechsleri Tucker sein können, jedoch sind die letzten drei Phytopathogene am häufigsten an Wurzelfäule im Bundesstaat Alagoas beteiligt (Abbildung 1) und in den meisten produzierenden Regionen. Informationen über die Fäulnis der Maniokwurzeln sind nach wie vor in erster Bedeutung und von größter Bedeutung, um als Instrument bei der Entscheidungsfindung über die beste $z u$ verwendende Strategie zu dienen (MASSOLA JR; BEDENDO, 2005; MUNIZ et al., 2006; NOTARO et al., 2013; SILVA, 2013; FUKUDA, Ich bin NICHT ZU SEIN OTSUBO, 2015).

Unter den Mikroorganismen, die Maniokwurzelfäule P. drechsleri und F. solani verursachen, sind sie aufgrund des Umfangs und der Schwere, die erhebliche Produktionsverluste verursachen, die wichtigsten. In dichten und versauerten Böden ist das Auftreten von Fusarium sp. häufiger, und eines der Merkmale dieser Krankheitserreger ist es, schäden am Stamm in der Nähe des Bodens durch Infektion der xylemischen Gefäße und folglich in die Zirkulation des Safts stören, wodurch indirekte Fäulnis der Wurzel in jeder Ursache der Pflanzenentwicklung verursacht. Im Gegensatz zu Phytophthora, deren Symptome in den Wurzeln in der Farbe hervorgerufen werden, und weich-Konsistenzfäule, die eine Flüssigkeit mit schlechtem Geruch ausstrahlen, bereits die Fäulnise von Fusarium sp., ohne offensichtliche 
Störungen im Gewebe mit Anzeichen von Dehydrierung und mumifiziertem Aussehen einer trockenen Fäulnis (MOURA; SILVA, 1997; POLTRONIERI et al., 2001; SILVA, ich werde nicht TEIXEIRAS, 2012).

Als Schutz- und Wurzelfäulebekämpfungsmaßnahmen wird physikalische und chemische Bodenbewirtschaftung eingesetzt, aber die Verwendung toleranter Sorten, die mit kulturellen Praktiken wie Fruchtfolge und Anbausystem in Verbindung gebracht werden, wurde positiv unterstützt. Nascimento Jénior (2015) betont, dass es von grundlegender Bedeutung ist, die edapho-klimatischen Bedingungen des Anbaus und den Einsatz geeigneter Techniken zu kennen, die vom Landwirt übernommen werden können, um eine wirtschaftliche Rendite innerhalb eines nachhaltigen Agrarsystems zu erzielen. Die alternative Kontrolle von Phytopathogenen mit Antagonisten, insbesondere Trichodermiearten, wurde bereits untersucht, da das weltweite Interesse an Produkten, die weniger umweltaggressiv sind, weltweit nicht bekannt ist (SOUSA et al., 2012; SILVA et al., 2014). Für Robbs (1992) und Barbosa; Meza (2009), der Wettbewerb um Nahrung und Antibiose, sind die Mechanismen, die von einigen Trichodermie-Arten am häufigsten verwendet werden und daher als ausgezeichnetes Biokontrollmittel angesehen werden.

Aus diesem Grund bestand das Ziel der vorliegenden Arbeit darin, eine bibliographische Überprüfung der wichtigsten Phytopathogene und des Potenzials des Trichoderma-Antagonismus durchzuführen, der mit Maniokwurzelfäule im Bundesstaat Alagoas verbunden ist.

\section{METHODIK}

Die vorliegende Studie besteht aus einer Literaturrecherche, die zwischen Dezember 2014 und März 2016 durchgeführt wurde, in der eine Konsultation zu wissenschaftlichen Artikeln durchgeführt wurde, die durch die Suche in der Datenbank von Google Academic, LILACS, MEDLINE, SciELO, Portal von Capes (Thesen und Dissertationen), Zeitschriften und Büchern ausgewählt wurde. Von den zahlreichen 
verwandten Artikeln wurden 84 verwendet. Als Deskriptoren verwendeten wir: Wurzelfäule, biologische Kontrolle von Phytopathogenen, Phytosanität.

\section{LITERATURKRITIK}

\subsection{CASSAVA-ANBAU}

Cassava, eine Kultur von großem Interesse für den brasilianischen Nordosten, insbesondere für den Staat Alagoas, ist eine mehrjährige, strauchige Pflanze, die zur Euphorbiaceous-Familie gehört, reich an Stärke, die in menschlichen und tierischen Lebensmitteln oder als Rohstoff für verschiedene Industrien verwendet wird (FRAIRE FILHO; BAHIA, 2015).

Nascimento Jénior (2015) betont die Vorteile dieser Kultur, aufgrund ihrer Rustikalität, niedrigen Kosten, Anpassung in Bezug auf das Klima, Boden und seine Ernährungseigenschaften, die mit der großen Ansammlung von Stärke in seinen Wurzeln, sowie Proteine und Aminosäuren in den Blättern zusammenhängen. Es ist eine Pflanze mit Ursprung in den tropischen Regionen Südamerikas, die aber als Orte ihrer Domestizierung Südostasien, Afrika und Tropisches Amerika hatte (NOTARO et al., 2013; SILVA, 2013; FRAIRE SON; BAHIA, 2015).

Im Jahr 2013 wurden weltweit 281.718.000 Tonnen Maniok produziert, wobei Brasilien mit 23.977.757 Tonnen der viertgrößte Produzent der Welt ist, hinter Nigeria, Indonesien und Thailand. Die nördlichen und nordöstlichen Regionen Brasiliens haben eine große Produktionsmenge und Anbaufläche ist im Süden, Südosten und Mittleren Westen, die mit dem höchsten landwirtschaftlichen Ertrag. Der Staat Para ist der größte nationale Produzent von Maniokwurzeln, gefolgt von Parané, Maranhéo und Bahia (IBGE, 2015).

In Alagoas sind die Regionen, die sich als Maniokproduzenten auszeichnen, die wilden, $70 \%$ der Gesamtproduktion des Staates (Arapiraca, Sao Sebastiéo und Campo Alegre), die Waldzone und die Nordküste, die erste Region, die mit der größten Anzahl von Produzenten, verantwortlich für 13,36 tha-1 und 240.448 Tonnen, nimmt 
den zweiten Platz in der Produktivität im Vergleich zu den anderen Staaten des Nordostens (CONAB 2013).

Notaro (2012) betonte, dass diese Kultur noch Familienbetrieb ist und sich rudimentär entwickelt, und das Hauptvermehrungsmittel ist vegetativ und Sorten von geringer genetischer Qualität werden immer noch angebaut. Seine Nützlichkeit ist mit dem Gehalt an Hydrocyansäure $(\mathrm{HCN})$ in Blättern und Wurzeln verbunden, die es als sanftmütig oder süß(<180 mg kg-1 de $\mathrm{HCN})$, auch als "Macaxeira" bekannt, für den Frischverzehr verwendet; Zwischenprodukte (180 - $300 \mathrm{mg} \mathrm{kg}^{-1}$ ) und Bitter- oder Wildprodukte (> $300 \mathrm{mg} \mathrm{kg-}^{-1}$ ) werden für die Herstellung von Tierfutter, Stärke, Mehl und Biokraftstoffen verwendet (OLIVEIRA et al., 2012).

Der größte Nutzen von Maniok ist in der Lebensmittelindustrie, vor allem in der Produktion von Stärke für den modifizierten Stärkemarkt bestimmt, die neben der regionalen Küche in Brot, Tapioka, Beijus, KaugummiKuchen und Tomboy Fuß; ist eines der Produkte aus dem Werk, das mehr Arbeit beschäftigt und einen hohen Wert in das verarbeitete Produkt hinzufügt, nach Nascimento Jénior (2015). Was die Verwendung für die Gewinnung von fermentierten Produkten in der Biokraftstoffindustrie betrifft, so hat sich man dinaals als eine gute Alternative bei der Herstellung von Ethanol erwiesen, da es laut Salla et al. (2010) niedrigere Energiekosten in der Produktion darstellte.

Laut Alves (2006) kann die Phänologie des Manioks zwischen 6 und 24 Monaten Anbau variieren, aber kommerziell sind es 12 bis 18 Monate für die Verarbeitung von Mehl und Stärke und zwischen 8 und 10 Monaten für den Tisch. Unter den in Alagoas gepflanzten Sorten sticht Rosinha hervor, deren Zyklus zwischen 9 und 10 Monaten im Trockenen variiert und unter bewässerten Systembedingungen für 7 bis 8 Monate erwartet werden kann. Diese Sorte kann drei Meter hoch werden, hält den Pflanzenstand am Ende der Ernte mit guter Produktion von Zweigen und Produktivität in 21 tha-1 (DINIZ et al., 2009). 
Nascimento Jénior (2015), berichtet in seiner Arbeit, dass auch mit der Rustikalität der Ernte notwendig ist, ist aufmerksam auf die adafo-klimatischen Bedingungen, dass Maniok produziert gut in fruchtbaren Böden, mit pH zwischen 5,5 bis 7,0 mit sandigen oder mittleren Texturklasse und gute natürliche Drainage. Der ideale klimatische Zustand liegt im Temperaturbereich zwischen $20^{\circ} \mathrm{C}$ und $27^{\circ} \mathrm{C}$ und kann in Umgebungen mit Temperaturen zwischen $16{ }^{\circ} \mathrm{C}$ und $38^{\circ} \mathrm{C}$ ermittelt werden; mit Niederschlägen zwischen 1000 und 1500 mm Jahr-1, in semiariden Regionen zwischen 500 und 700 mm Jahr-1; Höhen von 600 bis 800 Metern über dem Meeresspiegel und Lichtperiode von 12 Stunden Tag-1 (SILVA; ANDRADE, 2011; SILVA, 2013).

\subsection{KRANKHEITEN IN CASSAVA CROP}

Mehrere Faktoren können die Cassava-Produktion einschränken, wie das Auftreten von Insektenschädlingen und krankheitserregenden Phytopathogenen, was die Qualität und Entwicklung der Ernte in Brasilien und weltweit beeinträchtigt. Laut Nascimento Jénior (2015) ist Maniok anfällig für mehrere Phytopathogene, was zu schweren wirtschaftlichen Produktionseinbußen führt.

Unter den Von bedeutungsvollen Krankheiten stechen die Ursachen von Blattläsionen wie brauner Fleck (Cercosporidium henningsii) weißer Fleck (Phaeoramularia manihotis), brennende Blätter (Cercospora vicosae), schwarzer Fleck (C. manihobaea) und Anthraknose (Colletotrichum gloeosporioides f.sp. manihotis), die Photoassimilaten begrenzen (SILVA; ANDRADE, 2011; MORAIS et al., 2013; OLIVEIRA et al., 2013). Und Wurzelfäule, verursacht durch Pilze und Oomicetes, wie Lasiodiplodia sp., Pythium sp., Rosellinia sp., Fusarium sp., Neoscytalidium lignicola, Phytophthora drechsleri Tucker jedoch sind die letzten drei Phytopathogene am häufigsten an Wurzelfäule im Zustand von Alagoas beteiligt (Abbildung 1) und in den meisten produzierenden Regionen. Informationen über die Fäulnis der Maniokwurzeln sind nach wie vor in erster Bedeutung und von größter Bedeutung, um als Instrument bei der Entscheidungsfindung über die beste zu verwendende Strategie zu dienen 
(MASSOLA JR; BEDENDO, 2005; MUNIZ et al., 2006; NOTARO et al., 2013; SILVA, 2013; FUKUDA, Ich bin NICHT ZU SEIN OTSUBO, 2015).

Notaro et al., (2013) und Silva (2013) warnen vor den hohen sozioökonomischen Auswirkungen, die durch Maniok-Wurzelfäule in den Bundesstaaten Maranhéo, Para, Alagoas und Pernambuco verursacht werden, da dies erhebliche Produktivitätsverluste verursacht und die Flächen nicht mehr für die Anpflanzung während der gesamten Erntezyklen geeignet macht. Laut Nascimento Jénior (2015) sind Omyceto Phytophthora sp und der Pilz Fusarium sp. die Haupterreger der Kultur im Nordosten und verursachen Verluste zwischen 30 und 70\%. Der Autor stellt auch fest, dass unter günstigen Bedingungen die Krankheit 100\% Verlust verursachen kann.

Abbildung 1. Aspekt der Kolonien und Fortpflanzungsstrukturen von Phytopathogenen verursachenden Maniokfäule Isolate im Staat Alagoas: Fusarium sp. (A1-A6), Neoscytalidium sp. (B1-B6) und Phytophthora sp. (C1-C6). Kolonie Fusarium sp. (A1); Conidia (A2); konidiogene Zelle (A3-A6). Neoscytalidium sp. Kolonie (B1); Conidia (B2-B6). Phytophthora sp Kolonie (C1); Spomangio (C2); Chlamydosporen (C3 und C4); Spomangios (C5) und Sporantia in Reproduktion (C6).

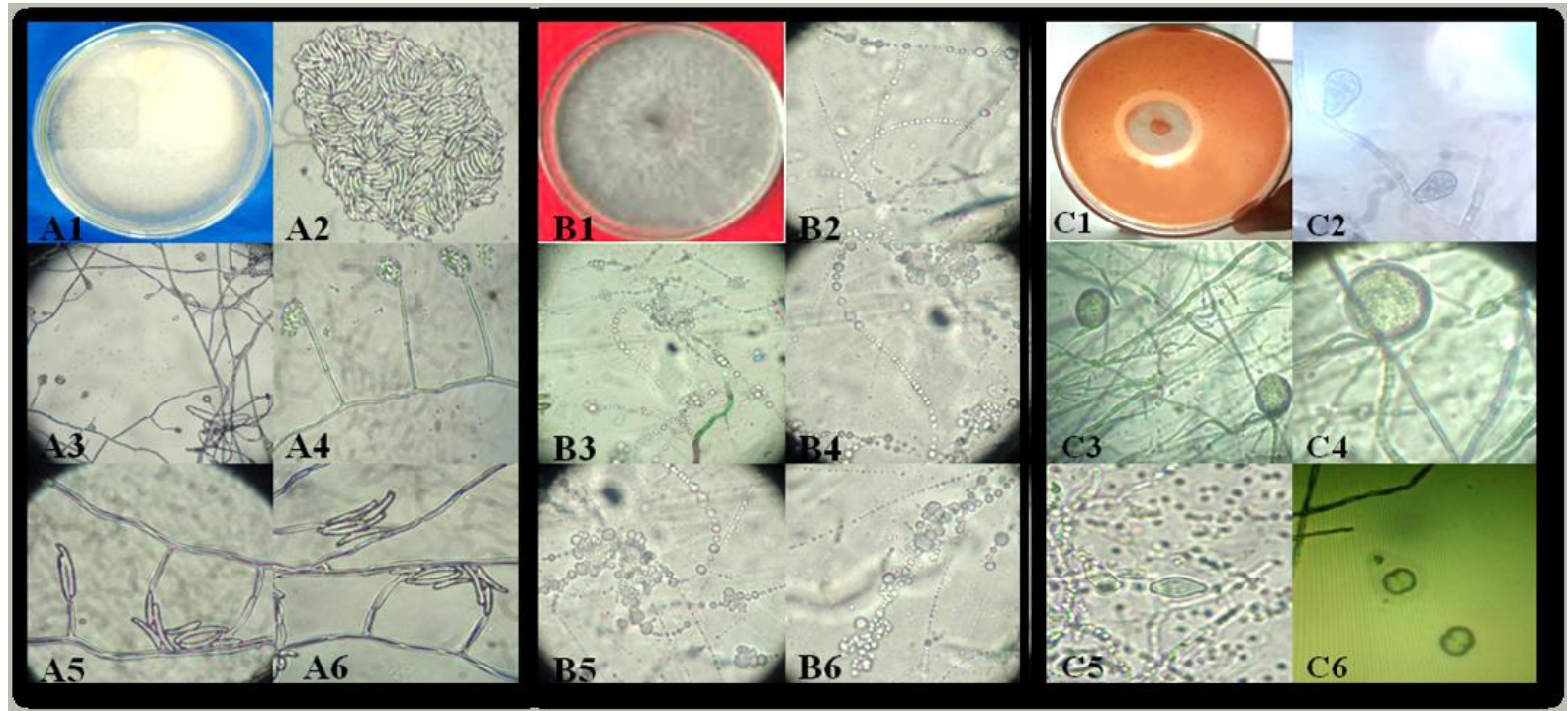

Fonte: SILVA e NASCIMENTO JÚNIOR (2016).

Disponível em: https://www.nucleodoconhecimento.com.br/agronomie-de/trichoderma- 
Die durch Fusarium sp. verursachte Fäulnis tritt in jungen und erwachsenen Pflanzen auf, mit Symptomen eines plötzlichen Welkens und schwerer Entlaubung, die in Bereichen lehmiger und schlecht entwässerter Böden potenziert werden können (SILVA; TEXEIRA, 2012). Diese Tatsache wurde bereits von Gomes berichtet; Leal (2003), der bei der Arbeit mit Maniok in den Bundesstaaten Sergipe, Bahia und Alagoas erklärt, dass Wurzelfäule die begrenzendste Krankheit der Kultur in der Region Nordosten ist, vor allem, wenn sie in Gebieten implantiert wird, die durch verdichteten Boden gebildet werden.

Die Phytopathogene, die die Fäulnis verursachen, haben deutliche Symptome. Die Autoren berichten, dass Phytophthora sp. in der Regel erwachsene Pflanzen angreift und "weiche" Fäulnis in den Wurzeln verursacht, mit sehr starkem Geruch, ähnlich dem von zerfallender organischer Substanz und grauer Färbung. Das Auftreten von Zeichen ist häufiger und sichtbar in den Wurzeln, aber es gibt Berichte über die Existenz von jungen Menschen, die Symptome an der Basis der Stämme von neu gezigen Pflanzen, verursacht Welken und Totaltod. Bei Trockenfäule, die durch Fusarium sp. verursacht wird, kommt es selten zu direkten Wurzelschäden, aber das Problem wird in jeder Phase der Pflanzenentwicklung verstärkt. (GOMES; LEAL, 2003; SILVA; TEXEIRA, 2012; NASCIMENTO JÚNIOR, 2015).

\subsection{DAS PHYTOPATHOGEN FUSARIUM}

Die Gattung Fusarium wurde 1809 von Link beschrieben. Die Vertreter dieser Gattung gehören zum Königreich Pilze, phylum Ascomycota, Klassen Eauscomycetes und Pyrenomyceten, bestellen Hypocreales, präsentiert 65 Arten, 55 Sorten und 22 besondere Formen, nach Wollenweber; Reinking (1935) ${ }^{1}$ zitiert von Ventura (1999). Es ist eines der wichtigsten Phytopathogene der Welt, mit einer breiten geographischen Verteilung, verteilt im Boden und in Verbindung mit Pflanzen. Mit kosmopolitischen und anderen Arten mit Vorkommen auf bestimmte Umgebungen beschränkt (VENTURA, 1999). Die meisten Arten sind saprobic, aber es gibt Vertreter des Fusarium-Komplexes, die Mykotoxine produzieren, die die Gesundheit von Mensch und Tier beeinträchtigen können (URBEN et al. 2009). Seifert (2006); 
Querales (2010) berichten, dass die Gattung Fusarium eine komplexe Gruppe ist, die an Arten wächst und taxonomische Kontroversen für Phytopathologen erzeugt.

Milanesi (2009), kommentiert, dass Fusarium sp., kann vor allem in Orten der tropischen und subtropischen Klimaauftreten auftreten, und hat eine große Fähigkeit, für lange Zeit im Boden außerhalb seiner Gastgeber zu überleben, durch die Bildung von Widerstandsstrukturen genannt chlamydospores. Drei Gruppen innerhalb der Gattung sind die spezifischsten Beispiele für diese Veränderungen, benannt nach der Teleomorph-Gruppe des Gibberella fujkuroi (Sawada) Wollenw-Komplexes, des Fusarium solani (Mart.) Sacc. und des F. graminearum-Komplexes.

Die Gattung kann Kolonien von Farben von violett, dunkle Purpura bis Orange präsentieren, aber die Dominanz ist von blasskolonie des schnellen Wachstums, luftige und diffuse Myzel (MARTINS, 2005). Puhalla (1981); Martins (2005); Agrios (2005), Bericht, dass Conidia-Strukturen (Mikro und Makro), Morphologie, Disposition, Vielfalt der Conidiophore und Chlamydosporen waren die Grundlage für die Identifizierung von Fusarium sp Arten. Klassische Taxonomen beschreiben das Vorhandensein von einzelligen und nicht inulektierten Mikrokonidien und häufigen multizellulären Makrokonidien mit Zellen mit nur einem Kern als genetisch identischen Vorläufer. Die sexuellen Stadien von Fusarium sind Ascomycetes; die sexuelle Sporen ist die Ascospore. Diejenigen, die in der Gattung Nectria zugeordnet sind, haben bizelluläre Askosporen.

Jede Zelle des bizellulären Askopors ist uninukleatiert und beide Kerne sind genetisch identisch. Andere, wie es bei Gibberella der Fall ist, bilden mehrzellige Ascosporen. Martins (2005) erwähnt, dass morphologische Eigenschaften von der Umwelt und der Ernährung beeinflusst werden, so dass Kulturmedien verwendet werden, um Mikroorganismen auszuwählen und sie zu gruppieren, basierend auf den Ernährungsbedürfnissen, die in der Kulturumgebung unter standardisierten Bedingungen zur Verfügung gestellt werden, um sie zu identifizieren. Aufgrund dieser Plastizität und Variationen der phänotypischen Eigenschaften, die in diesem Pilz 
gefunden werden, ist die Taxonomie, die nur auf morphologischen Konzepten basiert, nicht zuverlässig. (MARTINS, 2005; URBENS et al., 2009; QUERALES, 2010).

Die Identifizierung von Fusarium-Arten ist einer der ersten Schritte für ihre Untersuchung. Traditionell wurde es auf der Grundlage der Morphologie durchgeführt, seit der Veröffentlichung der Abhandlung der Gattung "Die Fusarien" im Jahr 1935 von Wollenweber; Reinking1. Andere Systeme wurden vorgeschlagen, lösten aber nicht das Problem der Artenidentifikation. Die Identifizierungsinstrumente wurden ausgebaut und umfassen den Einsatz optischer und elektronischer Mikroskopie, selektive und differenzielle Mittel, enzymatische Vergleiche, sekundäre Metaboliten sowie den Einsatz immunologischer und molekularer Technologien (LEAL-BERTIOLI, 1998; MARTINS 2005). O'Donnell et al., (2000); Summerell et al., (2003); Ottoni, (2008); Kommentar, dass mit dem Aufkommen molekularer Werkzeuge die Identifizierung von Fusarium auf der Morphologie, Biologie und Charakteristik der Art basiert, basierend auf sexueller Verträglichkeit, Phylogenie und Sequenzen von genomischen Regionen.

Seit Jahrzehnten verwendet die klassische Identifizierung morphologische Merkmale als Grundlage, hat aber Kontroversen ausgelöst, weil mehrere Taxonomen und Phytopathologen es für notwendig halten, andere Modelle zu verwenden, um die Art zu trennen, während andere glauben, dass diese Modelle die Taxonomie der Gattung beeinflussen. Bemerkenswert ist, dass die Praxis bei der Diagnose von Erregern von Krankheiten bei der Pflanzung der Einführung genetischer Modelle stärker an die von Mykologen durchgeführten Studien angepasst ist. Dennoch sind diese Veränderungen relevant, da es in einer Gattung sehr nahe Arten geben kann und dies zu Fehldiagnosen mit wichtigen praktischen Folgen führen kann (QUERALES, 2010).

Nach Notaro et al. (2013) Fusarium solani Math (Sacc) ist die Art, die häufig Wurzelfäule in Cassava cv branquinha im Bundesstaat Pernambuco verursacht. Diese Art produziert, in Kulturmedium, Luftmyzel, mit Farbe von weiß bis creme, bläulichbraun, wenn sporodochios vorhanden sind. Es produziert drei Arten von asexuellen Sporen: die slece-förmige Makrokonidie, mit ungefähren Abmessungen von 15-35 x 4- 
7 m mit 3-5 Septa; Mikroconidien, die Septum haben oder nicht, messen etwa 5-10 x 1-3 m und Chlamydosporen, die Hyalin, Globose, glatt bis rau sind, und zwar mit einer Größe von 6-10 m.

Der sexuelle Zustand des Pilzes ist Nectria haematococca (Ascomycete). Dieser Pilz überlebt auf infizierten kulturresten, Erde, Knollen und Samen und kann mehrere Jahre bestehen bleiben. Die idealen Bedingungen für Ihr Haus sind Temperaturen von 15 bis $25^{\circ} \mathrm{C}$, Luftfeuchtigkeit von 50 bis $75 \%$, Auftreten von Verletzungen, Anpflanzung in kontaminierten Böden und unzureichende Lagerung. Verletzungen des Wurzelsystems, insbesondere solche, die durch Nematoden verursacht werden, erleichtern das Eindringen des Pilzes in die Wurzeln der Wirte.

\subsection{KRANKHEITSBEKÄMPFUNG}

Da es sich um einen erregerer handelt, der vom Boden übertragen wird, wird seine Eliminierung nach der Installation im Pflanzbereich zu einer sehr schwierigen Aufgabe, und chemische Kontrollmaßnahmen gibt es praktisch nicht, außer vorbeugenden, wie die Verwendung von Saatgutbehandlungen oder anderen Vermehrungsmaterialien (LAZAROTTO et al., 2012). Die kulturelle Kontrolle, basierend auf der Prävention von Verletzungen während der Ernte und Lagerung, die Verwendung von gesundem Saatgut und Substrat frei von dem Erreger, nur einmal verwendet, die ausgewogene Anwendung von Nährstoffen, Effizienz in der Bewässerung, sowie Sorten, die Resistenzeigenschaften und die Behandlung von Stecklingen (Maniva) und Substrat mit Fungizid für die Aussaat bei der Infektion , kann Produktionsverluste minimieren.

Als Kontrollmaßnahme beruhte die effizienteste Maßnahme auf Forschungsarbeiten, die im Nordosten von Embrapa Cassava und Fruit And Agricultural Development Company (EMAGRO) durchgeführt wurden, die Verwendung von toleranter Sorte (Osso Duro, Cedinha, Bibiana, Klon 148/02, Aramaris und Kiriris), die mit Fruchtfolge und Pflanzsystem verbunden war, die Fäulnis um etwa $80 \%$ reduzierte (GOMES; LEAL, 2003; BETTIOL, 2009; LOBO J'NIOR et al., 2009; LAZAROTTO et al., 2012). 
Für die Kontrolle von Fusarium sp. sind die chemikalieDnnochonazol und Thiram bei der Behandlung von Boden und Saatgut aufgrund der dargestellten Effizienz am meisten zu empfehlen. Die chemische Kontrolle ist jedoch konstant geworden und mit dem unterschiedslosen Gebrauch sind ökologische Probleme entstanden, da diese Veränderungen in der Umwelt verursachen, die Phytopathogene mit Resistenz ento pathogen durch selektiven Druck, Das Auftreten von sekundären Ausbrüchen, Die Verringerung der Population nützlicher Mikroorganismen, aufgrund der Ablagerung von Resid, die sich im Boden, Wasser und Nahrung ansammelt, die sie schädlich machen, mit schädlicher Wirkung für Tiere und Menschen (BIZI , 2015).

Im Allgemeinen machen die hohen Kosten der Praxis der chemischen Kontrolle, die mit der Entstehung von Resistenzen gegen häufig verwendete Produkte verbunden sind, sowie das Verbot von Methylbromid die Entwicklung alternativer Kontrolltechniken erforderlich (BETTIOL, 2009; LOBO J'NIOR et al., 2009; LAZAROTTO et al., 2012). Für die biologische Pflanzenresistenz-Induktion ist eine vielversprechende Alternative, da sie natürliche Extrakte mit mikrobiellen und/oder induzierenden Eigenschaften verwendet (MORAES, 1992; STANGARLIN et al., 2008).

\subsection{BIOCONTROL VON PHYTOPATHOGENEn}

Der Versuch, die schädlichen Handlungen eines Erregers oder Inokulums zu reduzieren, war in der Landwirtschaft eine Konstante, und die Substitution von Agrochemikalien durch natürliche Produkte durch Unterdrückungs- oder Kontrolltätigkeiten war eine der praktikablen Alternativen, da die zurückgelassenen Rückstände aufgrund ihrer geringen Persistenz schneller von der Umwelt assimiliert und zersetzt werden (SOUZA, 2013; BIZI, 2015).

Ethur, (2006) und Dianese, (2007); die Bedeutung der Forschung im Bereich der biologischen Kontrolle, wie die Einführung antagonistischer Organismen in Umgebungen, in denen der Erreger Schäden verursacht, zu melden. Cancela (2015) stellt fest, dass die Dynamik der Wirkung der biologischen Kontrolle auf natürliche Weise auftritt und in der Regulierung der Anzahl der Pflanzen und Tiere durch 
natürliche Feinde besteht. Diese Strategie ist ein grundlegender Bestandteil des Gleichgewichts der Natur und der Art und Weise, wie chemische Kontrolle Vor- und Nachteile hat. Zu den Vorteilen gehört die Tatsache, dass es sich um eine ungiftige Maßnahme handelt, keine Unausgewogenheit verursacht, keine Kontraindikationen hat, eine umfassende und effiziente Kontrolle bietet, wenn es keine chemische Kontrolle gibt, weil sie aufgrund ihrer langsamen Wirkung mehr Technologie benötigt, schwer zu erwerben ist und nicht immer zu jeder Jahreszeit angewendet werden kann.

Die Aufrechterhaltung des Gleichgewichts im Agroökosystem ist eine Praxis der biologischen Kontrolle, so dass das Pathosystem keine signifikanten Schäden in der Biocontrolling-Wirkung nichtpathogener Organismen des Systems verursacht. Die Autoren berichten auch, dass Krankheit mehr ist als eine Pathogen-/WirtsInteraktionsbeziehung, da der Einfluss der Umwelt und auch eine Vielfalt nichtpathogener Organismen am Infektionsort am Infektionsort liegt, kann die Aggressivität des Erregers oder die Wirtsresistenz einschränken oder erhöhen. So interagieren Erreger, Wirt und Antagonist in den Komponenten der biologischen Kontrolle miteinander (BIZI, 2015; CANCELS 2015).

Ein begrenzender Faktor für die Verwendung biologischer Produkte ist die Verfügbarkeit dieser Kontrollmodalität auf dem Markt. Ein weiteres Problem ist, dass viele Naturprodukte nicht ordnungsgemäß für den kommerziellen Gebrauch registriert sind (SILVA et al., 2008). Das Vorhandensein des Phytopathogens stört die Pflanzenhomöostase, kann aber auch durch auslösende Abwehrmechanismen und/oder aktivierende Gene wirken, die Resistenzen der Wirtspflanze verleihen (AGRIOS, 2005). Michereff et al., (1993), erklären, dass der Erreger bestimmende Aktivitäten mit Wachstum, Infektiosität, Aggressivität, Virulenz und anderen Zuschreibungen des Erregers oder Prozesse hat, die Infektion, Entwicklung der Symptome und Fortpflanzung bestimmen. Die Wechselwirkung zwischen Antagonist, Wirt und Erreger tritt nicht in einer bestimmten Weise auf, sondern gegenseitig.

Mehrere Autoren haben bereits die Fähigkeit von Biocontrangers beschrieben, durch Wirkmechanismen wie Denkanstoß um Raum und Nährstoffe gegen Antibiose, 
Mykoparasitismus oder Hyperparasitismus, Raub, Induktion von Resistenzen in die Entwicklung des Phytopathogens einzugreifen und sich einzumischen, wodurch der Wettbewerbsvorteil in der Umwelt erhöht wird. Diese Mechanismen variieren je nach Wirts-Parasiten-Interaktion von Art zu Art und auch von Abstammung zu Abstammung innerhalb derselben Art. (CARVALHO, 2006; BETTIOL; MORANDI, 2009; BRITO et al., 2010; ZUCCHI, 2010; DIAS, 2011; MACHADO et al., 2012; BIZI, 2015; CANCELA, 2015; FIPKE, et al., 2015).

Viele Mikroorganismen haben ein hohes Biocontroller-Potenzial und die Gattung Trichoderma Pers. wurde in mehreren Studien verwendet. T. hamatum (Bonord.) Bainier, T. viride Pers., T. aureoviride Rifai, T. harzianum Rifai, T. koningii Oudem, T. pseudokoningii Rifai und T. longibrachiatum Rifai sind die am häufigsten zitierten (BETTIOL; GHINI, 2005). Ezziyyani et al., (2007) berichten, dass die Häufigkeit von Trichoderma spp., ist aufgrund seiner Vielseitigkeit entweder durch Wettbewerb, Hyperparasitismus und Antibiose zu Phytopathogenen wie Phytophthora, Fusarium und Neoscytalidium. Remuska \& Pria (2007) zur Bewertung der antagonistischen Wirkung von Trichoderma spp., bei der Kontrolle des myzelischen Wachstums von phytopathogenen Pilzen beobachtet, dass dies Antagonismus auf einige Wurzel Phytopathogene, wie Sclerotium rolfsii, Pythium aphanidermatum und Fusarium solani, wobei letztere Gattung als eine der wichtigsten Phytopathogene mit Cassava Wurzelfäule im Zustand von Pernambuco assoziiert beschrieben.

Hoffmann et al., (2015) berichteten, dass sie 80\% Kontrolle über Fusarium sp. hatten, mit Trichoderma spp Isolaten. Da Silva et al., (2011); Pereira (2013) bewies in seinen Werken eine große Hemmung des mycelialen Wachstums von $F$. solani durch Trichoderma spp Arten. Souza et al., (2015) hatte auch vielversprechende Ergebnisse mit Fusarium sp., und Bipolaris sp., mit dem gleichen Biocontroller. Weitere Studien von Bomfim et al., (2010); Dias (2011); Lazarotto et al., (2012); Santos et al., (2012); Marques et al., (2014), erzielte vielversprechende Ergebnisse mit guten bis ausgezeichneten Leistungen, in Bezug auf die Hemmung des mycelialen Wachstums von Phytopathogenen, die von innen getestet wurden. 


\subsection{TRICHODERMA BIOCONTROL STRATEGIES}

Die Gattung Trichodema, die zum Fungi Kingdom, phylum Ascomycota, Klassen Eauscomycetes und Pyrenomycetes, Order Hypocreales, Familie Hypocreaceae, Gattung Hypocrea (teleomorph) gehört. Dieser Pilz hat ein großes antagonistisches Potenzial und wird oft mit unterdrückenden Böden in Verbindung gebracht. (MELO, 1991). Nach Harman et al. (2004); Harman (2005), seine Verwendung kann andere Vorteile bieten: Zersetzung von organischer Materie, Wettbewerb mit einer schädlichen Mikroflora durch die Besiedlung der Rhizosphäre, Produktion von Antibiose und Induktion von Resistenz.

Trichoderma-Arten wurden bereits für die Bekämpfung verschiedener Krankheitserreger dokumentiert, wie Rhizoctonia solani Kühn, Sclerotium rolfsii Sacc., Sclerotina sclerotiorum (Lib.) von Bary, Fusarium spp. e Pythium spp. (MELO; AZEVEDO, 1998). Zahlreiche Trichodermiearten, darunter T. harzianum Hifai, wiesen eine hohe Rate an Parasitismus und antagonistischer Aktivität für Phytopathogene wie $S$. rolfsii, S. minor und $S$. sclerotiorum, $R$. solani und Pyricularia grisea Sacc auf. (ROLLAN et al., 1999; RONDÓN et al., 2007).

Laut Dos Santos (2010) präsentierte das Isolat von T. harzianum in vitro Antagonismus über den Pilz S. rolfsii; in der Lage, die Hyphen des Erregers zu hyperparasitisieren und um Raum und Nährstoff zu konkurrieren. Silva et al. (2013), die mit Isolaten von Trichodema spp., als biologisches Kontrollmittel von S. lignicola, beobachtete, dass zunächst das Isolat von Neoscytalidium sp. es war sehr vielversprechend, wenn mit Trichoderma spp. konfrontiert, aber als sie in der Petrischale gefunden wurden, Trichoderma spp., überlagerte die Krankheitserregerkolonie. Durch die Beobachtung der Region, in der sich der Antagonist mit Phytopathogenen überlappt, konnte ein Abbau des Hyphen des Erregers durch enzymatische Wirkung festgestellt werden, da er nur Sporen und Hyphen vom Antagonisten genau unterschieden wurde.

Für Robbs (1992) und Barbosa; Meza (2009), der Wettbewerb um Nahrung und Antibiose, sind die Mechanismen, die am häufigsten von Biokontrollmitteln verwendet 
werden, da Fortpflanzung und schnelle Kolonisierung Attribute von Organismen sind, die die verfügbaren Ressourcen effizienter und schneller nutzen und ihr Überleben sichern. Laut Ethur (2006) ist der Wettbewerb eine der Hauptstrategien von Trichodermie-Sp.-Isolaten, die dafür als Biocontroller eingesetzt werden.

Die Sekretion organischer Antibiotika (Antibiose), die von Mikroorganismen erzeugt werden, die selbst in geringer Konzentration in metabolische Aktivitäten eingreifen, Hemmung oder Stillstand von Wachstum und Sporulation, Verringerung der Sporenkeimung und Verzerrungen bei Hyphen und Endolyse, ob spezifisch oder breit bewertet, sind wichtige Mittel für die Effizienz von Antagonistenorganismen (LOBO JUNIOR; ABREU, 2000; BOMFIM, 2007; SOUZA, 2013). Laut Claydon et al. (1987) sind Antibiotika Produkte des Sekundärstoffwechsels ihrer Produzenten und können wichtiger sein, um andere Organismen zu hemmen als den Wettbewerb um Nährstoffe. Antibiose wurde auch von mehreren Forschern als einer der Wirkmechanismen der Trichoderma-Arten berichtet (BETTIOL, 1991; DIAS, 2011; CARVALHO FILHO, 2013). Gliotoxin, Viridin und Trichodermine sind einige der Substanzen, die von Trichoderma-Arten produziert werden, die die Entwicklung anderer Pilze hemmen können (Amorim et al., 2011). Ethur (2006) berichtete, dass Gliotoxin für die Hemmung der Keimung von Spomangios und das myceliale Wachstum von Pythium ultimum verantwortlich war.

Nach Bomfim et al., (2010) und Souza (2013), wird der Biocontroller erfolgreich sein, wenn es in der Lage ist, die Zellwand (bestehend aus Kohlenhydraten, wie Chitin), sowie Proteine und Lipide in signifikanten Mengen des Phytopathogens zu abbauen oder die Prozesse im Zusammenhang mit seiner Synthese zu unterbrechen. Daher ist die Produktion von Enzymen, die die Zellwandkomponenten von Phytopathogenen hydrolysieren, insbesondere Chitinasen und Glucanasen, ein häufiges Merkmal in vielen Biokontrollmitteln. Einige Vertreter des Schwiegersohns Trichoderma werden in Werken als ausgezeichneter Produzent von Cellulase und Chitinasen, Enzymen, die Cellulose und Chitin abbauen, zitiert (MELO, 1991; HARMAN et al., 2004). 
Der Mykoparasitismus, eine Situation, in der der antagonistische Mikroorganismus auf oder innerhalb des antagonisierten Pilzes lebt und sich auf seine Kosten ernährt und entwickelt, ist ein weiterer wichtiger Wirkmechanismus als Attribut eines guten Biocontrollers. Mykoparasitismus beinhaltet Antibiose und Kannibalismus durch die Wirkung von hydrolytischen Enzymen verursacht, wie Chitinasen, Glucanasen, Proteasen und Lipasen, die den Tod eines von innen verursachen, die somit als Nahrung für den Überlebenden dient (LOBO JUNIOR; ABREU, 2000; HARMAN, 2000; BOMFIM, 2007; BARRA et al., 2008; ZUCCHI, 2010).

Chitinasen und Glucanase sind direkt an den Wechselwirkungen des Mykoparasitismus zwischen Trichodermie-Arten und inren Wirten beteiligt, da die Mehrheit der phytopathogenen Pilze in ihrer Chitinzellwand besitzt, die in regelmäßig geordneten Schichten und als amorphe Füllung angeordnete 1,3-Glucane angeordnet sind. Chitinase wirkt zufällig und degradiert die biologischen Strukturen und Komponenten von Pilzzellen, setzt Oligomere frei, die Exoquitinasen induzieren, wodurch der mykoparasitische Angriff beginnt, indem die Oligosaccharide der phytopathogenen Zellwand hydrolysiert werden. (GIESE et al., 2003; MARCELLO, 2008; BAUERMEISTER et al., 2010).

Melo (1996) berichtet, dass nekrotropher Mykoparasitismus im Antagonismus für Phytopathogene, insbesondere solche mit Resistenzstrukturen, die als schwer von Mikroorganismen angegriffen werden können, wie Spros, Sclerotia, Chlamydosporen und Mikrosklerotie, effizient ist. Melo, melo. Azevedo (1998) besagt, dass Trichoderma sp. es hat Mykoparasiten-Eigenschaften, weil sie Phytopathogene lokalisieren und erkennen können, möglicherweise durch biochemische Reize, in Richtung Hyphen von anfälligen Pilzen wachsen, und wenn sie in Kontakt kommen, bilden sie Strukturen wie Appressorien, krümmen sich in vollem Umfang, durchdringen und verdauen sie. Die Gattung Trichoderma ist zweifellos ein ausgezeichnetes mikrobielles Kontrollmittel, da sie neben den oben beschriebenen Fähigkeiten wesentliche Eigenschaften aufweist: Umweltbelastung und geringe oder null leichte Vermehrung (SPIEGEL; CHET, 1998), Fähigkeit, in ungünstigen Umgebungen zu überleben, außer Populationen von Krankheitserregern, die im Boden leben (VINALE et al., 2008). 


\section{ABSCHLIEßENDE ÜBERLEGUNGEN}

Interspezifische ökologische Beziehungen sind Ereignisse, die auf verschiedenen Ebenen in der Natur auftreten und meist aus dämonischen Formen bestehen, wobei der Zustand der Beziehung zwischen Mikroorganismen, die Konkurrenz, Antibiose und Parasitismus am häufigsten sind. So verursachen krankheitserregende Mikroorganismen in Pflanzen nicht nur wirtschaftliche Verluste, sondern entwickeln sich gleichzeitig und verursachen so Schäden aus der Anfangsphase der Pflanzenentwicklung. Traditionelle Methoden der Phytopathogenbekämpfung wurden verwendet, die Anwendung von chemischen Pestiziden und Fungiziden, war auch eine sehr häufige Form, die große Probleme aufgrund von Umweltauswirkungen verursachen.

Eine der vielversprechendsten Möglichkeiten, um eine Verringerung des Einsatzes dieser Agrochemikalien zu erreichen, oder sogar eine zukünftige Substitution, biologische Kontrolle durch die zahlreichen Biocontroller wie Pilze der Gattung Trichoderma, die effizient sind und keine zerstörerischen Umweltauswirkungen verursachen.

\section{REFERENZEN}

AGRIOS, G.N. Plant pathology. Amsterdam : Elsevier Academic Press. 5.ed, 2005. 922p.

ALVES, A., A., C.. Fisiologia da mandioca. In: Aspectos socioeconômicos e agronômicos da mandioca. EMBRAPA Mandioca e Fruticultura Tropical, Cruz das Almas, 2006., 817p.

AMORIM, L.; REZENDE, J. A. M.; BERGAMIN FILHO, A. Manual de Fitopatologia. Piracicaba: Agronômica Ceres. v. 1,4. Ed, p. 383 -387, 2011.

BARBOSA, R.J.F.; MEZA, C.L.S. Antagonismo in vitro de Trichoderma harzianum rifai sobre Fusarium oxysporum schlecht $f$. sp passiflorae en maracuyá 
(Passiflora edulis sims var. flavicarpa) del municipio zona bananera colombiana. Rev. Fac. Nal. Agr. Medellín, v.62, n.1, p.4743-4748, 2009.

BARRA, V. R.; ROMEIRO, R.S.; FERRAZ, H.G.M.; MACAGNAN, D.; SILVA, H. S. A.; MOURA, A. B.; HALFELD-VIEIRA, B. A; MENDONÇA, H. L.; VIEIRA JÚNIOR, J. R. Potencialidade antagonística em alguns procariotas agentes de biocontrole de enfermidades de plantas. Ver. Summa Phytopathologica, v.34, n.2, p.121-126, 2008.

BAUERMEISTER, A.; REZENDE, M. I.; GIESE, E. C.; DEKKER, R. F. H.; BARBOSA, A. M. 1,3-Glucanases Fúngicas: produção e aplicações biotecnológicas. LONDRINA SP. rev. Semina: Ciências Exatas e Tecnológicas, , v. 31, n. 2, p. 75-86, jul./dez. 2010. http://www.uel.br/seer/index.php/semexatas/article/view/5249

BETTIOL, W. Controle biológico de doencas do filoplano. In: BETTIOL, W., org. Controle biológico de doencas de plantas. Jaguariuna: EMBRAPA-CNPDA, 1991. p.33-52 (EMBRAPA-CNPDA. Documentos, 15).

BETTIOL, W.: MORANDI, M.A.B. Controle Biológico de Doenças de Plantas no Brasil. In: BETTIOL, W.: MORANDI, M.A.B. (Org). Biocontrole de Doenças de Plantas: uso e perspectivas. Jaguariúna - SP: Embrapa Meio Ambiente, p. 7-14, 2009.

BETTIOL, W.; GHINI, R. Solos Supressivos. In: MICHEREFF,S. J.; ANDRADE, D. E. G. T.; MENEZES, M. (Eds.) Ecologia e Manejo de patógenos radiculares em solos tropicais. Recife:UFRPE, Imprensa Universitária, 2005. cap.6, p. 125-143.

BIZI, R. M., Utilização de fungos no Controle Biológico de Doenças. http://www.floresta.ufpr.br/alias//pf/public_html/contbio03.html.> Acesso em 16/11/2015.

BOMFIM, M. P., Antagonismo in vitro e in vivo de Trichoderma spp. a Rhizopus stolonifer em maracujazeiro amarelo. Vitória da Conquista BA, 2007. 74f.: Dissertação de Mestrado. Universidade Estadual do Sudoeste da Bahia. 2007 
BOMFIM, M. P., SÃO JOSÉ, A. R., REBOUÇAS, T. N. H., ALMEIDA, S. S. D., SOUZA, I. V. B., \& DIAS, N. O . Avaliação antagônica in vitro e in vivo de Trichoderma spp. a Rhizopusstolonifer em maracujazeiro amarelo. Summa Phytopathologyca, v.36, n.1, p. 61-67, 2010.

BRITO, F. S.; MILLER, P. R. M., STADNIK, M. Presença de Trichoderma spp em composto e suas características para o controle de fitopatógenos. Revista. Bras. de Agroecologia, Porto Alegre , p. 43-53. 2010.

CANCELA, K. C., Controle Biológico Florestal Conceitos, Terminologia, Descrição e Caracterização de Agente. Proteção florestal Disponível em <http://www.floresta.ufpr.br/alias/pf/public_html/contbio01.html> Acesso em 16/11/2015.

CARVALHO FILHO, M. R., Relações filogenéticas, identificação e potencial de uso de isolados de Trichoderma no controle do mofo branco e como promotores de crescimento do feijoeiro. Brasília-DF, 2013. 123f. Tese de Doutorado. Programa de Pós-graduação em Fitopatologia, Universidade de Brasília. Brasília. 2013.

CARVALHO, A. C., Bioprospecção de isolados de Trichoderma stromaticum para o controle biológico da Vassoura-de-Bruxa do Cacaueiro. 2006. 82f. Dissertação de Mestrado. Universidade Estadual de Santa Cruz. 2006.

CONAB- CLAYDON, N.; ALLAN, M.; ITANSON, J.R.; AVENT, A. G. Antifungal alkyl pyrones of Trichoderma harzianum. Transactions of the British Mycological Society, v.88, p.503-513, 1987. COMPANHIA NACIONAL DE ABASTECIMENTO. Perspectivas para a agropecuária. Conab, Brasília , v.1, 2013., 154p.

DA SILVA, D. C. V., TIAGO, P. V., \& DE SOUZA-MOTTA, C. M. . Isolamento e seleção de fungos filamentosos do solo de sistemas agroflorestais do Município de Bom Jardim (PE) com base na capacidade de produção de enzimas hidrolíticas1. Revista Brasil. Bot, 34(4), 607-610. 2011 
DIANESE, A. de C. Variabilidade e controle de Phytophthora palmivora (Podridão-do-pé) e controle da varíola (Aperisporium caricae) do mamoeiro (Carica papaya). 2007. 109f. Tese Doutorado - Universidade de Brasília, Brasília. 2007

DIAS, P. P., Controle biológico de fitopatógenos de solo por meio de isolados de fungos do gênero Trichoderma e sua contribuição no crescimento de plantas. 2011. 101 f. Tese Doutorado. Universidade Federal Rural do Rio de Janeiro. Seropédica - Rio de Janeiro. 2011

DINIZ, M. de S.. Efeito da manipueira na adubação da mandioca. Revista Raízes e Amidos Tropicais, v. 5, 2009.

DOS SANTOS, J. et al. Atividade hiperparasíticade Trichoderma spp. sobre escleródios de Sclerotinia sclerotiorum em solo. In: SEMINÁRIO DE INICIAÇÃO CIENTÍFICA TECNOLÓGICA,7,2010.Anais...EPAMIG,2010.Online.Disponívelem:http://www.epa mig.br/index.phpoption=com_docman\&task=cat_view\&gid=121\&dir=DESC\&order=da te\&limit=10\&limitstart=10.Acesso em Dezembro 2015

ETHUR, L.Z. Dinâmica populacional e ação de Trichoderma no controle de fusariose em mudas de tomateiro e pepino. 2006. 154p. Tese (Doutorado em Fitopatologia) - Universidade Federal de Santa Maria, Santa Maria-RS. 2006.

EZZIYYANI, M.; REQUENA, M. E.; EGEA-GILABERT, C.; CANDELA, M. E. Biological Control of Phytophthora Root Rot of Pepper Using Trichoderma harzianum and Streptomyces rochei in Combination. Journal of Phytopathology , [S.I], v.155, n.6, p. 342-349, 2007

FAO. Faostatidtics Database. Disponivel em <https://www.fao.org.br/FAO_Brf2mpu3a.asp> Acesso em Dezembro de 2015. 
FIPKE, G. M; PAZINI, J.B.; ETHU,L. Z. Antagonismo de isolados de Trichoderma spp. ao Sclerotinia sclerotiorum em diferentes temperaturas. Magistra, Cruz das Almas BA, V. 27, N.1, p. 23 - 32, Jan./Mar. 2015

FUKUDA C.; OTSUBO A.A. Cultivo da mandioca na região centro sul do Brasil. Embrapa Mandioca e $\quad$ Fruticultura. HTTP://sistemasdeproducao.cnptia.embrapa.br/FontesHTML/Mandioca/mandioca_c entrosul/doenc s.htm . Acessado em 16 de julho de 2015.

FRAIFE FILHO G. A., BAHIA J. J. S., Mandioca. Ceplac-Cepec-BA. http://www.ceplac.gov.br/radar/mandioca.htm . Acessado Dezembro de 2015.

GIESE, E. C.; BARBOSA, A. M.; SILVA M.L.C. Glucanases Fúngica: Produção e aplicações das -1,3 e -1,6 glucanases. Revista Biotecnologia Ciência e Desenvolvimento, Brasília-DF, № 30 , P.97-104.. jan/jun 2003.

GOMES, J. C.; LEAL, E. C., Cultivo da Mandioca para a Região dos Tabuleiros Costeiros. In.: Podridão Radicular. Embrapa Mandioca e Fruticultura. Sistemas de Produção, 11. ISSN 1678-8796 Versão eletrônica/Jan/2003. https://sistemasdeproducao.cnptia.embrapa.br/FontesHTML/Mandioca/mandioca_ta bcosteiros/doencas.htm . Acesso 13/12/2015.

HOFFMANN, C. A. et al.. Potencial de antagonismo de isolados de Trichoderma sp. contra o isolados de Fusarium sp., in vitro. Revista Verde de Agroecologia e Desenvolvimento Sustentável, v. 10, n. 1, p. 236-242, 2015.

HARMAN, G. E. The Nature and Application of Biocontrol Microbes II: Trichoderma spp. Overview of Mechanisms and Uses of Trichoderma spp. Phytopathology, St Paul, v. 96, p. 190-194. 2005

HARMAN, G. E. Myth and dogmas of biocontrol changes in perceptions derived from research on Trichoderma harzianum T-22. Plant Disease, St. Paul, v. 84, p.377-393, 2000. 
HARMAN, G.E. HOWELL, C. R., VITERBO, A., CHET, I., \& LORITO, M. Trichoderma species-opportunistic, avirulent plant symbionts. Nature Reviews Microbiology. V.2, p. 43-56. 2004.

IBGE - Instituto Brasileiro de Geografia e estatística. PAS - Pesquisa Anual de Serviços, 2015. [online]Disponível na internet via WWW URL: http://www.ibge.gov.br/home/. Arquivo consultado Dezembro de 2015.

LAZAROTTO, M.; BOVOLINI, M. P.; MACIEL, C. G.; MUNIZ, M. F. B. Seleção in vitro de Isolados de Trichoderma spp. com Potencial de Antagonismo a Isolados Patogênicos de Fusarium spp. In: XVI Simpósio De Ensino, Pesquisa E Extensão, Unifra, v. 3, 2012.

LEAL- BERTIOLI, S. C. DE M. O enfoque molecular na sistemática de fungos. Revisão Anual de Patologia de Plantas. V.6. p. 197-230, 1998

LOBO JÚNIOR, M. e ABREU, M.S. Inibição do crescimento micelial de Sclerotinia sclerotiorum por metabólitos voláteis produzidos por alguns antagonistas em diferentes temperaturas e pH's. Ciência Agrotécnica, Lavras, v.24, p.521-526, 2000.

LOBO JUNIOR, M.; BRANDÃO, R. S.; CORRÊA, C. A.; GÖRGEN, C. A.; CIVARDI, E. A.; OLIVEIRA, P. de. Uso de braquiárias para o manejo de doenças causadas por patógenos habitantes do solo.Santo Antônio de Goiás: Embrapa Arroz e Feijão, 2009. 8 p. (Embrapa Arroz e Feijão. Comunicado técnico, 183).

MACHADO, D. F. M.; PARZIANELLO, F. R.; SILVA, A. C.F. e ANTONIOLLI, Z. I. Trichoderma no Brasil: o fungo e o bioagente. Revista de Ciências Agrárias, v.35, n.1, p. 274-288, 2012.

MARCELLO, C. M. Avaliação da expressão e caracterização de uma exo-b-1,3glucanase envolvida no mecanismo de micoparasitismo de Trichoderma asperellum. Brasília-DF, 2008. 89f. Tese de Doutorado. Laboratório de Enzimologia 
Departamento de Biologia Celular do Instituto de Biologia da Universidade de Brasília - UnB. Brasília - DF. 2008.

MARQUES, H. I. P.; SILVA, M. B.; MARQUES, M. D. P.; RODRIGUES, R. C.; RIBEIRO, P. R. C. C. Inibição do crescimento micelial de Trichoderma harzianum por fertilizantes líquidos. Enciclopédia Biosfera, v. 10, n. 18, p. 2040, 2014.

MARTINS, M. K.. Variabilidade genética de isolados de Fusarium spp. e estudo da interação com a planta hospedeira. 2005. 110f. Diss. Tese (Doutorado em Agronomia)-Escola Superior de Agricultura" Luiz de Queiroz", Universidade de São Paulo, SP.[Links], 2005

MASSOLA JR, N.S.; BEDENDO, I. P.. Doenças da mandioca. In: KIMATI, H. et al. Manual de fitopatologia: Doenças das plantas cultivadas;. 4ª Ed.vol. 2, p. 340-341 São Paulo: Agronômica Ceres, 2005

MELO, I. S. Potencialidades da utilização de Trichoderma spp. no controle biológico de doenças de plantas. In: BETTIOL, W. (Ed.). Controle biológico de doenças de plantas. Jaguariúna: CNPDA/EMBRAPA, 1991. p. 135-156.

MELO, I. S.; AZEVEDO, J. L. Ecologia Microbiana. Jaguariúna: Embrapa DNPMA, p. 393-419, 1998.

MELO, I.S. Trichoderma e Gliocladium como bioprotetores de plantas. Revis. Anu. Patol. Plantas v. 4, p. 261-295. 1996.

MENEZES, J. P., LUPATINI, M., ANTONIOLLI, Z. I., BLUME, E., JUNGES, E., \& MANZONI, C. G. Variabilidade genética na região its do rDNA de isolados de trichoderma spp.(Biocontrolador) e Fusarium oxysporum f. sp. Chrysanthemi. Ciência e Agrotecnologia, 34(1), 132-139.2010

MICHEREFF, S.J.; MENEZES, M.; MARIANO, R.L.R. Potencial de Trichoderma para o controle da antracnose do sorgo. Fitopatologia Brasileira, Brasília, v. 18, p. 392-398, 1993. 
MILANESI, P.M. Caracterização, toxicidade e patogenicidade de Fusarium spp. em genótipos de soja em sistema plantio direto. 2009. 91 p. Dissertação (Mestrado em Agronomia) - Universidade Federal de Santa Maria, Santa Maria, 2009.

MORAES, W. B. C. Controle alternativo de fitopatógenos. Pesquisa Agropecuária Brasileira, v. 27, n. 13, p. 175-190, 1992.

MORAIS, M. dos S.; NASCIMENTO, L. C do; MOREIRA, K. A.; SILVA, M. da; CAVALCANTI, N. T. D. O. Levantamento e avaliação da incidência das doenças da mandioca no estado da Paraíba. Summa Phytopathologica, v. 39, n. 3, p. 204. 2013.

MOURA, G. de M.; SILVA, M. D. O. da. Avaliação de resistência de cultivares de mandioca à podridão de raízes. Embrapa-CPAF/AC. Comunicado Técnico, 4p. Rio Branco 1997.

MUNIZ J. N, RUFFINO-NETTO A, YAMAMURA M, VILLA TCS, ARCENCIO R. Aspectos epidemiológicos da coinfecção tuberculose e vírus da imunodeficiência humana em Ribeirão Preto (SP), de 1998 a 2003. J. Bras. Pneumol., 32(6):529-34, 2006

NASCIMENTO JÚNIOR, N. A. Efeito da casca de mandioca no controle da podridão radicular causada por Phytophthora sp. em mandioca de mesa (Manihot esculenta Crantz) var. Rosinha em ambiente irrigado. Rio LargoAL,2015.82f. Tese de Doutorado. (Programa de Pós-Graduação em Proteção de Plantas da Universidade Federal de Alagoas. Alagoas. 2015.

NOTARO, K. A. MEDEIROS, E. V., SILVA, C. A. D., \& Barros, J. A. Prospecção de fitopatógenos associados á podridão radicular da mandioca em Pernambuco, Brasil. Biosci. J., Uberlândia, v. 29, n. 5, p. 1832-1839, 2013.

NOTARO, K. A. Prospecção de fitopatógenos e caracterização de solos arenosos envolvidos na supressividade ou conducividade da podridão radicular da 
mandioca, causada por Neoscytalidium lignicola/ Krystal de Alcantara Notaro. Garanhuns-PE, 2012. 111f. Dissertação (PROGRAMA DE PÓS-GRADUAÇÃO EM PRODUÇÃO AGRíCOLA). Universidade Federal Rural de Pernambuco - Unidade Acadêmica de Garanhuns, 2012.

O`DONNELL, K.; NIRENBERG, H.I.; AOKI, T.; CIGELNIK, E. A multigene phylogeny of the Gibberella fujikoroi species complex: detection of additional phylogenetically distinct species.Mycoscience, Tokyo, v. 41, p. 61-78, 2000.

OLIVEIRA, L. G. SILVA, A.C.S; SILVA, R. L. ,LIMA, D. W. S \& LIMA, G; COSTA. Atividade proteolítica de fungos de solo fitopatogênicos ao Feijão-Caupi [Vigna unguiculata (L.) Walp.] In: III CONAC Congresso Nacional de Feijão-Caupi. Pernambuco. Resumo expandido. 4p. 22-24 de abril Recife Pernambuco 2013.

OLIVEIRA, N. T; J. M. A., UCHÔA, S. C. P., RODRIGUES, G. S., MELVILLE, C. C., \& de ALBUQUERQUE, J. D. A. A.. Caracterização e identificação de clones de mandioca produzidas em Roraima para o consumo in natura. Revista Agro@mbiente On-line, v. 5, n. 3, p. 188-193, 2012.

OTTONI, R.J. Analise da incidência de Fusarium spp. toxigenicos e de níveis de fumonisinas em grãos ardidos de milho hibrido. 2008. 54 p. Dissertação (Mestrado em Microbiologia Agrícola) - Escola Superior de Agricultura "Luiz de Queiroz", Universidade de São Paulo, Piracicaba, 2008.

PEREIRA, C O.F. Estudo da patogenicidade e controle biológico de Fusarium sp. Com Trichoderma sp. 2013.74f. Dissertação. Universidade de Caxias do Sul. 2013.

PUHALLA, J.E. Genetic considerations of the genus Fusarium. In: Nelson, P.E.; Toussoun, T.A.; Cook, R.J. (Ed) Fusarium: diseases, biology, and taxonomy. Pennsylvania: Pennsylvania State University, v.27, p.291-305, 1981. 
QUERALES, P. J.. Caracterização morfológica e genética de Fusarium spp. isolados de sementes e associados à podridão do colmo de milho (Zea mays L.). Diss. Escola Superior de Agricultura "Luiz de Queiroz, 2010.

REMUSKA, A.C.; DELLA PRIA, M. Efeito de Bacillus truringiensis e Trichoderma sp. no crescimento de fungos fitopatogênicos. Publication UEPG: Ciências Exatas e da Terra, Agrárias e Engenharia, Ponta Grossa, v.13, n.3, p.31-36, 2007.

ROBBS, F. C. Controle Biológico de doenças em plantas. In: NETO, A. M. A.; BARAN, C. L. (ed.). Manual de Controle Biológico. Rio de Janeiro: Lidador. 1992. p.4651.

ROLLAN, MONACO C, NICO A. Efecto de la tempratura sobre la interaccion invitr o entre especies de Tricihoderma y Sclerotinia sclerotiorum, S. minor y Sclerotium rolfsii. Invest. Agr.: Prod. Prot. Veg. 14:1-16. 1999.

RONDÓN T.R, GUTIÉRREZ GR, ZAYAS A.D.P, PÉREZ LA, CUTIÑO Y.L. Efectividad in vitro de Trichoderma harzianum Rifai para el biocontrol de Rhizoctonia solani Kühn y Pyricularia Grisea sacc. aislados en el cultivo del arroz (Oryza sativa I.). Fitosanidad 11: 29-34.2007.

SALLA, D. A., CABELLO, C. Análise energética de sistemas de produção de etanol de mandioca, cana-de-açúcar e milho. Revista Energia na Agricultura. Botucatu, vol. 25, n.2, p.32-53., 2010.

SANTOS, C. C., OLIVEIRA, F. A. de; SANTOS, M. S. dos; TALAMINI, V.; FERREIRA, J. M. S.; SANTOS, F. J. dos., Influência de Trichoderma spp. sobre o crescimento micelial de Thielaviopsis paradoxa. Scientia Plena 8, 047309 www.scientiaplena.org.br . 2012

SEIFERT, K.A. Enhancing the relevance of fungal taxonomy to plant pathology: phylogenetics, molecular diagnostics, and long-term memory1. Symposium 
contribution. Canadian Journal of Plant Pathology, Otawa, v. 28, p. S280-S287, 2006.

SILVA, A. N.; AZEVEDO, G. B.; ROCHA-SOBRINHO, G. G.; NOVAES, Q. S.; Efeito de produtos químicos e de Trichoderma spp. no controle de Fusarium solani do maracujazeiro. Interciencia, v. 6, p. 398-403, 2014.

SILVA, C. A. D., DE MEDEIROS, E. V., BEZERRA, C. B., DE MORAIS; SILVA, W., DE BARROS, J. A., \& DOS SANTOS, U. J. Interferência da incorporação de matéria orgânica no solo no controle da podridão negra da mandioca, causada por Scytalidium lignicola. Biosci. J., Uberlândia, v. 29, n. 6 , p. 1823-1831, Nov./Dec. 2013.

SILVA, C. A. D., Prospecção em fitopatógenicos e avaliação de fontes de matéria orgânica sobre a supressividade da podridão radicular da mandioca. GaranhunsPE, 2013. 77 f. Dissertação (Mestrado em Produção Agrícola). Universidade Federal Rural de Pernambuco - Unidade Acadêmica de Garanhuns, 2013.

SILVA, H. S. A.; ANDRADE, E. C. Impacto potencial das mudanças climáticas sobre as doenças da mandioca no Brasil. In: Impactos das mudanças climáticas sobre doenças de importantes culturas no Brasil. Jaguariúna: Embrapa Meio Ambiente, p. 263-272, 2011.

SILVA, J. L.; TEIXEIRA, R. N. V. Esporulação e crescimento micelial de Fusarium solani em diferentes meios de cultura e regimes de luminosidade. Revista Agrombiente On-line,v.6,n.1,p.47-52, 2012. Disponível em: <http://revista.ufrr.br/index.php/agroambiente/article/view/604>

SILVA, K. S.; REBOUÇAS, T. N. H.; BOMFIM, M. P.; SILVA, D. S.; SÃO JOSÉ, A. R.; BENETT, C. G. S. Atividade antagônica in vitro de isolados de Trichoderma spp. ao fungo Phytophthora citrophthora. Londrina. Semina: Ciências Agrárias, v. 29, n. 4, p. 749-754, out./dez. 2008. 
SILVA, M.B.; NICOLI, A. COSTA, A.S.V.; BRASILEIRO, B.G.; JAMAL, C.M., SILVA, C. A.; PAULA JÚNIOR, T. J.; TEIXEIRA, H. Ação antimicrobiana de extratos de plantas medicinais sobre espécies fitopatogênicas de fungos do gênero Colletotrichum. Revista Brasileira de Plantas Medicinais, Botucatu, v.10, n.3, p.5760, 2008.

SOUSA, R.M.S.; SERRA, I.M.R.S.; MELO, T.A. Effect of essential oils as an alternative in the control of Colletotrichum gloesporioides in pepper.Summa Phytopathologica, v.38, n.1, p.42-47, 2012.

SOUZA, J. R., Potencialidade de fungicida e agente biológico no controle de requeima do tomateiro. 2013. 63f. Dissertação. Universidade Estadual do Sudoeste da Bahia, Vitória da Conquista. Bahia - BR. 2013.

SOUZA, K. de A.;VAZ, M. C. A.; ALVES, G. C. S.; PAZ -LIMA, M. L.. Confronto in vitro de isolados de Trichoderma spp. com fungos filamentosos. 2p. IV Congresso Estadual de Iniciação Científica do IF Goiano 21 a 24 de setembro de 2015.

SPIEGEL, Y.; CHET, I. Evolution of Trichoderma spp. as a biocontrol agent against soilborne fungi and plant parasitic nematodes in Israel. Integrated Pest Management Review, v. 03, p. 167-175, 1998.

STANGARLIN, J.R. et al. Controle de doenças de plantas por extratos de origem vegetal. Revisão Anual de Patologia de Plantas, v.16, p.265-304, 2008.

URBEN, A.F., et al., Curso taxonomia de Fusarium. Embrapa recursos genéticos e biotecnologia. Brasilia-DF: Embrapa informação tecnólogica, 2009.

VENTURA, J. A. Taxonomia de Fusarium e seus segregados: I- história, meios e procedimentos de cultivo. Revisão Anual de Patologia de Plantas, Passo Fundo, v. 7, p. 271-298, 1999. 
VINALE F, SIVASITHAMPARAM, K., GHISALBERTI, E. L., MARRA, R., WOO, S. L., \& LORITO, M.. Trichoderma-plant-pathogen interactions. Soil Biology and Biochemistry, v. 40, p.1-10.2008.

ZUCCHI, F. O. Trichoderma sp. em áreas agrícolas visando o controle de doenças fúngicas de raízes de plantas cultivadas. Campinas SP. JV Biotecnologia. 15p. 2010.

\section{ANHANG - FOOTNOTE-REFERENZ}

1. WOLLENWEBER, H. W.; REINKING, O. A.. Die fusarien: ihre beschreibung, schadwirkung und bekämpfung. Berlin: P. Parey, 1935

Eingesandt: Mai 2020.

Genehmigt: Juni 2020. 